\title{
Adequação de objetos de aprendizagem da disciplina de Redes de Computadores para a inserção de acessibilidade
}

\author{
Alisson Renan Svaigen ${ }^{1}$, Lailla Milainny Siqueira Bine ${ }^{1}$, \\ Luciana Andreia Fondazzi Martimiano ${ }^{1}$
}

${ }^{1}$ Departamento de Informática - Universidade Estadual de Maringá (UEM) Maringá - PR - Brasil

alisson.svg@gmail.com, lailla.milainny@gmail.com, lafmartimiano@uem.br

\begin{abstract}
The teaching-learning process is increasingly related to technology. Teachers seek alternative tools to improve quality of education and an option are the animations. To use animations as learning objects, it is necessary to adapt them, promoting the inclusion of students with special needs. This paper presents the initial stages of a developing research of a online learning object's repository and a mobile application, already developed to Computer Networks course, for accessibility's inclusion. The preliminaries results are the adequacy of these tools, making it easier future accessibility inclusions.
\end{abstract}

Resumo. O processo de ensino-aprendizagem está cada vez mais relacionado com a tecnologia. Os docentes buscam ferramentas alternativas para melhorar a qualidade do ensino, sendo uma opção as animações. Ao utilizar animações como objetos de aprendizagem, é necessário que elas sejam adequadas a todos os alunos, promovendo a inclusão de discentes com necessidades especiais. Este artigo apresenta as etapas iniciais de uma pesquisa em desenvolvimento de adequação de um repositório online de objetos de aprendizagem e um aplicativo para dispositivo móvel, já desenvolvidos para a disciplina de Redes de Computadores, para inclusão de acessibilidade. Os resultados preliminares são a adequação dessas ferramentas, facilitando inclusões de acessibilidade futuras.

\section{Introdução}

O ensino de disciplinas de graduação por parte dos docentes se torna um trabalho cada vez mais árduo devido aos avanços tecnológicos e ao perfil do discente que ingressa na Universidade. Os métodos de ensino-aprendizagem necessitam unir teoria à prática e manter o foco do aluno no conteúdo apresentado [de Souza 2011]. Para enfrentar os desafios apontados, se fazem úteis ferramentas auxiliares de ensino, como por exemplo, animações, simuladores e imagens. Além das ferramentas, deve-se avaliar os meios de disponibilização das mesmas, como por exemplo, a web, sistemas desktop e aplicativos para dispositivos móveis.

No Departamento de Informática da Universidade Estadual de Maringá foram desenvolvidos, em anos anteriores, trabalhos relacionados à utilização de animações como ferramentas de ensino-aprendizagem para o ensino da disciplina de Redes de Computadores por [de Souza 2011], [Tanamati 2012], [Bueno 2013] e [de Oliveira 2014], os quais 
V Congresso Brasileiro de Informática na Educação (CBIE 2016)

Anais do XXVII Simpósio Brasileiro de Informática na Educação (SBIE 2016)

culminaram na criação e disponibilização para o corpo discente de um repositório de objetos de aprendizagem online e de um aplicativo para dispositivos móveis.

Objetos de aprendizagem são entidades digitais pautadas na web que visam aprimorar o aprendizado guiando, acrescentando e ampliando conceitos específicos para influenciar o processo cognitivo [Wiley 2000]. Entre as entidades que podem ser consideradas objetos de aprendizagem estão as animações.

Um problema a ser considerado é que as ferramentas desenvolvidas não possuíam características de acessibilidade. Segundo os dados do Censo demográfico do IBGE (2010), 35,7 milhões de pessoas se declararam como deficientes visuais, sendo essa a deficiência mais presente, segundo o censo. Diante do fato que destaca o grande número de deficientes no país, uma perspectiva ideal seria oferecer soluções de aprendizagem igualitárias para todas as pessoas, sem distinção ou segregações[Lima and Tavares 2015].

Dessa maneira, caracteriza-se a inserção de acessibilidade nesses meios. Segundo [Torres et al. 2002], para o desenvolvimento de ferramentas com acessibilidade, a informação apresentada deve estar disponível e sem prejuízos a todos os usuários de maneira autônoma, independentemente das suas possíveis limitações. Visando suprir esse problema, foi elaborada uma linha de pesquisa de inclusão de acessibilidade nas ferramentas já existentes.

Esse artigo tem como objetivo apresentar as etapas até então desenvolvidas de uma pesquisa ainda em andamento da adequação para inclusão de acessibilidade - a saber, itens de acessibilidade audiovisual - nas animações do repositório de objetos de aprendizagem e do aplicativo para dispositivos móveis, promovendo a inclusão de pessoas com necessidades especiais para a utilização dessas ferramentas. As etapas foram compostas, de maneira geral, no estudo das diretrizes de acessibilidade internacionais e nacionais, no estudo das ferramentas a serem utilizadas e na metodologia de inserção de acessibilidade.

O artigo está organizado da forma que segue: esta seção apresenta a introdução e a contextualização do tema tratado neste artigo; na Seção 2 tem-se a apresentação do conceito de animações atrelado ao process de ensino-aprendizagem; na Seção 3 tem-se a descrição dos conceitos de acessibilidade; na Seção 4 são descritas as ferramentas e a metodologia utilizadas; na Seção 5, os resultados das primeiras etapas da inclusão de acessibilidade são descritos; na Seção 6 têm-se as considerações finais e os trabalhos futuros.

\section{Animações como ferramentas de auxílio no processo de ensino-aprendizagem}

Segundo [Santos 2012], os discentes contemporâneos, em sua maioria, possuem uma fidelização à utilização de tecnologias nas atividades do cotidiano e, indutivamente, nas atividades escolares. Assim, espera-se que os docentes se utilizem de tecnologias atuais para a diversificação do ensino e motivação do aluno, como por exemplo, a utilização de animações.

[Santana et al. 2009] utilizaram as animações como ferramenta para o ensino da disciplina de Estruturas de Dados, no qual concluíram que as animações devem ser utilizadas conjuntamente com as aulas tradicionais, sendo um material atrativo e interativo para o aluno. Já o trabalho de [Coura et al. 2006] avalia uma série de sistemas para construção 
V Congresso Brasileiro de Informática na Educação (CBIE 2016)

Anais do XXVII Simpósio Brasileiro de Informática na Educação (SBIE 2016)

de animações utilizando Programação por Demonstração, concluindo que as ferramentas são válidas e atingem seus objetivos.

No trabalho de [Urquiza-Fuentes and Velazquez-Iturbide 2012] são apresentadas duas abordagens de uso educacional de animações, uma construtiva e uma ativa, que são comparadas com o método tradicional de ensino. Os autores avaliaram que as abordagens não se fazem tão importantes para uso em assuntos simples, todavia, quando há um nível maior de complexidade (como é o caso da disciplina de Redes de Computadores), se torna necessário o apoio das animações.

\section{Acessibilidade e diretrizes de desenvolvimento}

A educação inclusiva (a que promove acessibilidade) é, segundo [Pansanato et al. 2012], uma das questões que desafiam os sistemas educacionais devido a uma série de necessidades, dentre as quais podem-se destacar a falta de formação específica do professor para tratar destas questões, bem como a falta de apoio na literatura. De acordo com [Boscarioli et al. 2015], o censo de 2011 do MEC/INEP apontou que dos mais de 8 milhões de alunos matriculados no ensino superior, cerca de $29033(0,32 \%)$ possuíam algum tipo de necessidade educacional especial.

As definições e questões de acessibilidade são garantidas pela lei $\mathrm{n}^{0} 10098$, de 19 de dezembro de 2000 e atualizada pela Lei 13146, de 6 de julho de 2015, a qual diz que acessibilidade é a "possibilidade e condição de alcance para utilização, com segurança e autonomia, de (...) informação e comunicação, inclusive seus sistemas e tecnologias, (...), por pessoa com deficiência ou com mobilidade reduzida"[Brasil 2015]. Para que ferramentas com acessibilidade sejam desenvolvidas, é necessário seguir recomendações de órgãos competentes, como é o caso das diretrizes WCAG[W3C 2014] e eMAG [Brasil 2014].

As diretrizes WCAG (Web Content Accessibility Guidelines) são promovidas pela W3C, o consórcio internacional entre organizações que desenvolvem os padrões Web. Constitui-se especificamente de 12 diretrizes, sendo elas divididas em quatro princípios: perceptível; operável; compreensível; e robusto. Já as diretrizes eMAG (Modelo de acessibilidade em governo eletrônico) são um conjunto de recomendações do governo federal brasileiro para promover a inclusão social e digital dos portadores de necessidades especiais, as quais são separadas em seis seções: marcação; comportamento; conteúdo/informação; apresentação/design; multimídia; e formulário. As recomendações eMAG são consideradas uma especialização da WCAG.

\section{Materiais e métodos}

Esta Seção apresenta os materiais e os métodos utilizados ao decorrer da pesquisa. São apresentadas inicialmente as ferramentas utilizadas, que possuem as animações já desenvolvidas. Após, é apresentada a metodologia aplicada para a inserção dos itens de acessibilidade nas animações dessas ferramentas.

\subsection{Ferramentas utilizadas}

Os principais materiais envolvidos nessa pesquisa são as duas ferramentas já existentes, que possuem as animações como objetos de aprendizagem, a serem adequadas para a inserção de acessibilidade, descritas nas seções a seguir. 
V Congresso Brasileiro de Informática na Educação (CBIE 2016)

Anais do XXVII Simpósio Brasileiro de Informática na Educação (SBIE 2016)

\subsubsection{Repositório de objetos de aprendizagem online}

O repositório foi desenvolvido utilizando tecnologias características para desenvolvimento web, a saber: HTML5, CSS, JavaScript e PHP. As animações foram desenvolvidas utilizando-se a tag Canvas, provida pelo HTML5, aliada ao JavaScript. A seguir, são listadas as animações presentes no repositório online:

- O Modelo Híbrido: Animação sobre o processo de encapsulamento e desencapsulamento realizado durante o envio de uma mensagem;

- O processo de Three-way Handshake do TCP (Transmission Control Protocol): Animação que mostra o processo de estabelecimento de conexão do protocolo TCP;

- O Protocolo Aloha Puro: Animação que mostra o funcionamento do protocolo Aloha, que resolve problemas de alocação de canais sem fio na camada de enlace;

- O Protocolo Ethernet: Animação sobre o funcionamento do protocolo de enlace mais utilizado nas redes com fio;

- TCP - número de sequência e de reconhecimento: Animação que explica dois importantes campos do cabeçalho do TCP.

- DNS: Animação que busca explicar o serviço de nomes da Internet utilizado para realizar a tradução de endereços IP;

- Roteamento Hierárquico: Animação que mostra o funcionamento do roteamento intra e inter AS (Autonomous Systems) e seus protocolos;

- Subredes: Animação que mostra a importância da utilização da máscara de rede para criação de subredes lógicas.

\subsubsection{Aplicativo para dispositivos móveis}

O aplicativo para dispositivos móveis foi inicialmente formulado e desenvolvido por [de Oliveira 2014]. O aplicativo tem como principais características ser internacionalizável e portável às diversas plataformas, por exemplo, Android, iOS e Windows Phone. Tal aspecto se deve às tecnologias envolvidas no projeto: Apache Cordova, Ionic Framework, GreenSock Animation Platform e AngularJS.

Antes do início desta pesquisa, constavam três animações no aplicativo, a saber:

- Three-way Handshake: Animação que mostra o processo de estabelecimento de conexão do protocolo TCP;

- Protocolo Aloha Puro: Animação que mostra o funcionamento do protocolo Aloha, que resolve problemas de alocação de canais sem fio na camada de enlace;

- TCP - número de sequência e de reconhecimento: Animação que explica dois importantes campos do cabeçalho do protocolo TCP.

\subsection{Metodologia aplicada}

Para a inserção dos itens de acessibilidade nas ferramentas utilizadas, buscou-se elaborar uma metodologia para que, ao término, se atendesse às recomendações eMAG e WCAG, desde que aplicáveis às ferramentas em questão. Devido às particularidades tecnológicas e arquiteturais de cada ferramenta, a metodologia aplicada é focada em cada ferramenta, que são apresentadas nas seções a seguir. 
V Congresso Brasileiro de Informática na Educação (CBIE 2016)

Anais do XXVII Simpósio Brasileiro de Informática na Educação (SBIE 2016)

\subsubsection{Metodologia da inserção de acessibilidade no Repositório de objetos de apren- dizagem Online}

Inicialmente, foi realizado um estudo do estado do repositório online, considerando estrutura do código e técnicas utilizadas no desenvolvimento. Alguns problemas foram encontrados como: as animações não estavam no mesmo padrão e possuíam problemas de usabilidade.

Visando a inclusão da acessibilidade e a partir dos problemas encontrados, foi definida a metodologia a seguir, em que os itens 1 e 2 são etapas "pré-inclusão"que foram necessárias para um bom andamento do projeto.

1. Correção dos problemas de usabilidade: Essa etapa permitiu a correção de alguns problemas de usabilidade na manipulação da animação pelo usuário, como por exemplo, a não existência do botão "Voltar";

2. Padronização das animações: Dois trabalhos anteriores foram responsáveis pela criação das animações no Repositório Online [Tanamati 2012] e [Bueno 2013]. Essa etapa proporcionou a padronização da estrutura das animações visando facilitar as etapas seguintes;

3. Inserção do áudio nas animações: Essa etapa buscou dar início a inclusão de acessibilidade áudio visual nas animações e possui as seguintes sub-etapas:

- Implementação do controle do som: Nessa primeira etapa foi realizada a preparação da animação para receber o áudio;

- Descrição textual das animações: As animações são, muitas vezes, dependentes de imagens para que seu contexto seja entendido. Esse fato implica que o áudio da animação deve conter também descrições mais detalhadas das imagens e outros fatores necessários para que um aluno com deficiência visual consiga acompanhá-la. Assim, essa etapa tem como foco a descrição das animações;

- Gravação do áudio das animações: Essa etapa tem como objetivo gravar as descrições textuais das animações;

- Sincronização do áudio nas animações: Essa etapa insere o áudio nas animações a fim de permitir a sua utilização por deficientes visuais;

\subsubsection{Metodologia de inserção de acessibilidade no aplicativo para dispositivos móveis}

Primeiramente, foi realizada uma avaliação geral da estrutura do aplicativo e um comparativo entre as animações do aplicativo com àquelas presentes no repositório online. Neste comparativo, foi avaliado que era necessário a implementação de cinco animações no aplicativo para que ambas as ferramentas tivessem o mesmo conteúdo em termos de tópicos da disciplina de Redes de Computadores.

Com relação à estrutura, foi constatado que o aplicativo não era responsivo, fato baseado na análise de que as animações foram projetadas para uma resolução de tela estática de 350x600 pixels, prejudicando a apresentação das mesmas em outros tipos de resolução. Conjuntamente com este problema, foi apurado que os ícones utilizados nas 
V Congresso Brasileiro de Informática na Educação (CBIE 2016)

Anais do XXVII Simpósio Brasileiro de Informática na Educação (SBIE 2016)

animações não possuíam formato vetorial, o que também poderia causar perda de qualidade dependendo da resolução de tela do dispositivo. Foi ainda avaliado que as animações não possuíam características de usabilidade para permitir que o usuário tivesse controle temporal sobre a animação.

Tendo em vista tais constatações, a metodologia seguida para a inserção de acessibilidade foi definida como a que segue:

1. Adaptação das animações do aplicativo para um formato responsivo: desenvolver funções e padrões de responsividade a serem aplicadas nas animações, bem como padronizar as imagens utilizadas em formato vetorial;

2. Desenvolvimento das cinco animações restantes, com relação ao repositório online;

3. Inserção de usabilidade: desenvolver um modelo de animação que suporte usabilidade, permitindo ao usuário um controle temporal sobre a animação;

4. Inserção de acessibilidade audiovisual: inserir no modelo desenvolvido no item anterior a acessibilidade audiovisual, por meio da descrição das animações;

5. Avaliação do aplicativo: realizar uma avaliação do aplicativo tanto com discentes com deficiencia visual como com discentes sem deficiencia.

\section{Resultados}

Nas seções a seguir são apresentados os resultados obtidos no repositório de objetos de aprendizagem online e no aplicativo para dispositivos móveis.

\subsection{Resultados obtidos no repositório de objetos de aprendizagem online}

As animações no Repositório possuíam um controle nada usual, o que acarretava em uma dificuldade de usuários comuns utilizarem a animação. Levando isso em consideração, optou-se por realizar a implementação da função "retroceder um passo"na animação, caracterizando a correção de problemas de usabilidade.

Uma animação é uma sequência de passos que são construídos e inseridos em uma pilha. A técnica utilizada na criação das animações é conhecida como flip-book, que consiste na troca rápida de imagens em curto período de tempo [Bueno 2013]. As partes dinâmicas das animações presentes no repositório utilizam essa técnica, ou seja, é realizada uma troca de várias imagens e cada imagem de um trecho dinâmico realiza uma chamada automática da próxima imagem.

Para a implementação da função "voltar", esse fator era um problema, pois ao retroceder um passo, ele se auto executava e avançava para imagem seguinte. A partir disso, foi criado um bloco de animação, assim o trecho dinâmico como um todo é inserido como um único passo na pilha. Em alguns casos, objetos não persistentes eram utilizados, assim durante um retrocesso esses objetos não eram redesenhados. De forma semelhante, foi criada uma estrutura de bloco para redesenhar partes do background não persistentes. Com esses problemas resolvidos, foi possível realizar a implementação do botão "Voltar". Também foi implementada uma função Javascript para acesso dos botões "Voltar"e "Avançar"por meio do teclado.

Após a correção de alguns problemas de usabilidade, verificou-se a necessidade de realizar uma padronização no código das animações. Como já mencionado, as animações 
V Congresso Brasileiro de Informática na Educação (CBIE 2016)

Anais do XXVII Simpósio Brasileiro de Informática na Educação (SBIE 2016)

disponíveis no repositório foram desenvolvidas por dois trabalhos anteriores. Assim, as animações desenvolvidas não estavam padronizadas. Para facilitar a implementação da acessibilidade, as animações foram padronizadas no modelo criado por [Bueno 2013]. Esse modelo foi escolhido, pois apresentava uma estrutura genérica mais adequada para as animações. Na Figura 1 é possível visualizar o antes (A) e depois (B) das adaptações realizadas na animação intitulada "Protocolo Aloha".

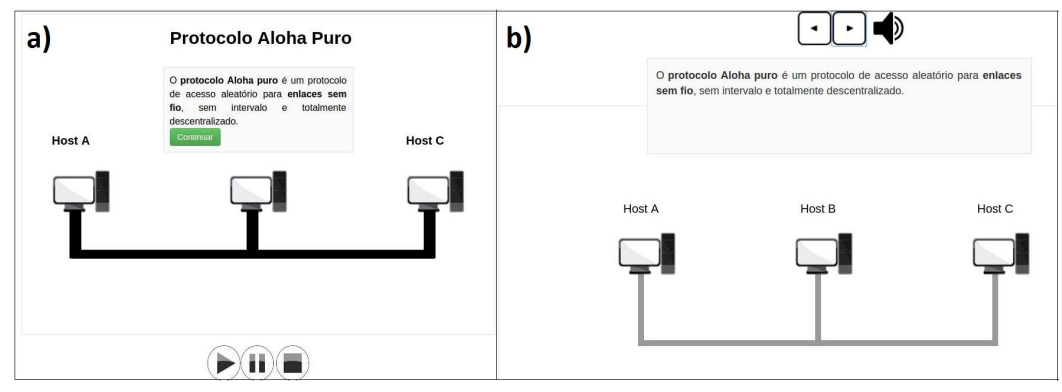

Figura 1. Animação "Protocolo Aloha" mostrando o antes(A) e depois(B) das adaptações.

Realizar a padronização é um meio de facilitar as etapas seguinte para a inserção de acessibilidade em si. As adaptações foram realizadas nas seguinte animações: o processo de Three-way Handshake do TCP; O Protocolo Aloha Puro; O Protocolo Ethernet; O Modelo Híbrido; TCP - número de sequência e de reconhecimento. As implementações realizadas foram baseadas nos algoritmos genéricos já existentes, no entanto, alguns métodos foram criados considerando as particularidades de cada animação que são descritos a seguir:

- MessageAnimationImg(): método que permite desenhar na tela um objeto ao mesmo tempo em que está ocorrendo uma movimentação de outro objeto;

- MessageAnimationImgAndShine(): método que permite simular o efeito do objeto piscar na tela ao mesmo tempo em que está ocorrendo uma movimentação de outro objeto;

- Increase(): método que permite realizar a movimentação do aumento de tamanho de um objeto;

- DecreaseLeft(): método que permite realizar a movimentação de diminuição de tamanho de um objeto e em seguida uma translação para a esquerda;

- DecreaseRight(): método que permite realizar a movimentação de diminuição de tamanho de um objeto e em seguida uma translação para a direita.

Após a etapa "pré-inclusão"de acessibilidade foi realizada a implementação de um botão para o controle do áudio seguindo a recomendação 5.4 do eMAG [Brasil 2014], que trata da importância do controle de som. Inicialmente, para teste, apenas uma animação recebeu sua descrição em áudio. A animação escolhida foi a intitulada "Roteamento Hierárquico". Para a inserção do áudio foi necessário realizar as seguintes etapas:

- Implementação do controle do som: Nessa primeira etapa foi realizada a preparação da animação para receber o áudio. Para a animação funcionar corretamente, foi implementada uma estrutura para que o som mantivesse a ordem correta, mesmo com a função "Voltar", já implementada nas animações. A estrutura escolhida foi uma pilha, pois é a mesma estrutura utilizada nos passos das animações, facilitando o controle conjunto dos mesmos; 
V Congresso Brasileiro de Informática na Educação (CBIE 2016)

Anais do XXVII Simpósio Brasileiro de Informática na Educação (SBIE 2016)

- Descrição textual da animação: Nessa etapa foi realizada a descrição da animação. As animações possuem imagens, tabelas e etapas dinâmicas que precisavam ser descritas, além do conteúdo em si para que o aluno possa compreender a animação;

- Gravação do áudio: A descrição foi gravada utilizando o sintetizador de voz eSpeak;

- Inserção do som na animação: Para inserir o áudio na animação, cada trecho foi ordenado com o seu passo correspondente e inserido na estrutura criada.

\subsection{Resultados obtidos no aplicativo para dispositivos móveis}

Considerando a metodologia proposta para a inserção de acessibilidade no aplicativo, a primeira adaptação realizada foi a substituição de todos as imagens do aplicativo para o formato vetorial (para aqueles que não se apresentavam nesse formato). Solucionado este problema, as animações responsivas foram refeitas em formato responsivo, ou seja, adaptáveis para qualquer resolução de dispositivo.

Com as animações no formato responsivo, foram desenvolvidas as demais animações presentes no repositório. Ao longo do desenvolvimento, percebeu-se que algumas animações possuíam muitos elementos em tela, o que dificultaria a organização em uma resolução de dispositivo móvel e prejudicaria também o entendimento por parte do usuário. Dessa maneira, essas animações não foram inseridas no aplicativo. As novas animações desenvolvidas foram: O Modelo Híbrido; Protocolo Intra-AS: RIP; Protocolo Inter-AS: BGP; Protocolo Ethernet.

A inserção de usabilidade foi realizada por meio da inclusão de "checkpoints"de orientação, que são portadas nas popups de informação. Com isso, o usuário passa a ter controle sobre o progresso da animação, podendo avançar ou retroceder em etapas. Foi também incluída a possibilidade do usuário reiniciar a animação ao final da mesma, bem como voltar para o menu de animações. Em todas as animações desenvolvidas foram inseridos os itens de usabilidade. A Figura 2 apresenta três popups de checkpoints desenvolvidas.

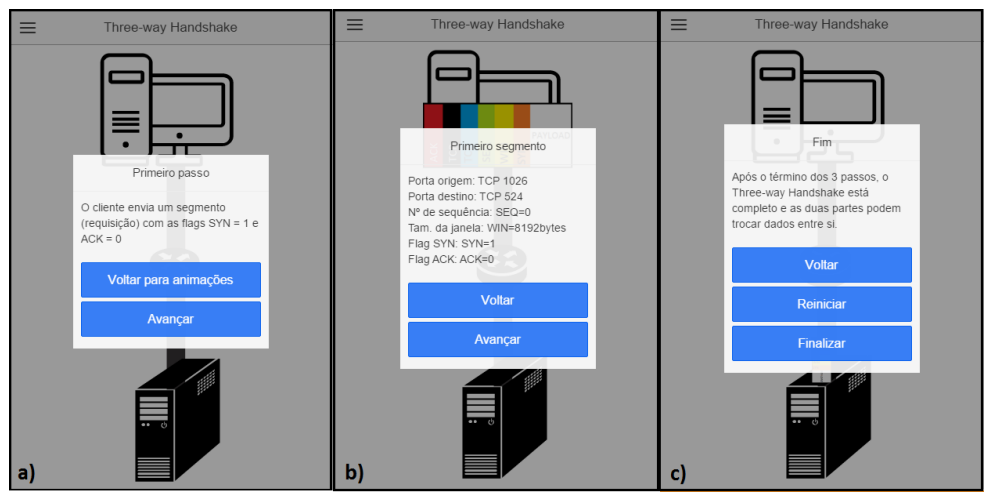

Figura 2. Modelos de popups de checkpoints implementados

O checkpoint a) é utilizado para o início da animação, permitindo ao usuário avançá-la ou retornar para o menu principal de animações. O checkpoint b) é utilizada para fluxo geral das animações, quando o usuário pode avançar ou retroceder para um checkpoint anterior. Já o checkpoint c) é utilizado ao fim da animação, quando o usuário 
V Congresso Brasileiro de Informática na Educação (CBIE 2016)

Anais do XXVII Simpósio Brasileiro de Informática na Educação (SBIE 2016)

tem o controle para voltar um passo, reiniciar a animação ou então finalizá-la e voltar para o menu principal de animações.

Devido ao tempo gasto nas etapas iniciais do projeto, principalmente no desenvolvimento das animações que se faziam presentes no repositório de objetos de aprendizagem, não foi realizada a implementação da acessibilidade audiovisual nas animações, até o momento em que este artigo foi redigido. Consequentemente, também não foi formulado e aplicada a avaliação a ser realizada. Como a pesquisa está em andamento, espera-se realizar tais etapas de forma plena.

\section{Conclusões}

Este trabalho apresentou o desenvolvimento das etapas até então desenvolvidas de inclusão de acessibilidade nas animações do repositório de objetos de aprendizagem online e no aplicativo para dispositivos móveis, culminando no início da adequação dessas ferramentas para utilização por pessoas com necessidades especiais. Baseado nas diretrizes nacionais e internacionais de acessibilidade, foram planejadas e apresentadas etapas para o desenvolvimento do trabalho.

Considerando que esta pesquisa utiliza ferramentas desenvolvidas em pesquisas anteriores, podemos destacar a dificuldade da inserção de elementos de acessibilidade, pois, durante o processo inicial de implementação das animações, não foi levada em conta a necessidade de incluir esses elementos. Esse fato acarretou em etapas "pré-inclusão" da acessibilidade que não haviam sido planejadas inicialmente, como a padronização das animações no repositório, a adequação do formato responsivo das imagens no dispositivo móvel e as correções de usabilidade nas duas vertentes do trabalho.

Os principais resultados apresentados neste trabalho são: a readequação estrutural das ferramentas para inclusão de usabilidade; o desenvolvimento de novas animações no aplicativo; a inserção de acessibilidade audiovisual em algumas animações do repositório. Tais resultados atendem as diretrizes 1.1, 1.2, 1.3, 1.4, 2.2 e 4.1 WCAG [W3C 2014]. Quanto às recomendações eMAG [Brasil 2014], já são atendidas as recomendações 1.4, $1.8,2.6,2.7,3.1,3.3,3.11,4.1,4.3,5.3,5.4$ e 5.5. Com tais recomendações sendo atendidas, proporciona-se uma maior qualidade estrutural para que a acessibilidade seja promovida.

As próximas etapas a serem realizadas são: inserir a acessibilidade audiovisual nas animações do aplicativo e finalizar as restantes do repositório; realizar as avaliações recomendadas pelas diretrizes de acessibilidade, provendo validade científica para a pesquisa desenvolvida; e disponibilizar as ferramentas.

\section{Referências}

Boscarioli, C., Galante, G., Oyamada, M. S., Zara, R. A., and Villwock, R. (2015). Aluno surdo na ciência da computação. 23 WEI - Workshop sobre Educação em Computação. Recife, Pernambuco.

Brasil (2014). eMAG. Ministério do Planejamento, Orçamento e Gestão, Secretaria de Logística e Tecnologia da Informação, $3^{\mathrm{a}}$ edition. 
V Congresso Brasileiro de Informática na Educação (CBIE 2016)

Anais do XXVII Simpósio Brasileiro de Informática na Educação (SBIE 2016)

Brasil (2015). http://www.planalto.gov.br/ccivil_03/ _Ato2015-2018/2015/Lei/L13146.htm\#art112. Lei n ${ }^{\circ} 13.146$, de 06 de julho de 2015 .

Bueno, D. C. (2013). Um repositório de objetos de aprendizagem para apoiar o ensino da disciplina de redes de computadores. Monografia (Graduação). Departamento de Informática - Universidade Estadual de Maringá.

Coura, D. P., Di lorio, V. O., Lima, A. G., Oliveira, A. P., and Andrade, M. V. A. (2006). [acm press vii brazilian symposium - natal, rn, brazil (2006.11.19-2006.11.22)] proceedings of vii brazilian symposium on human factors in computing systems - ihc '06 animações através de programação por demonstração.

de Oliveira, J. R. (2014). Uso de animações para o ensino de redes de computadores em dispositivos móveis. Monografia (Graduação). Departamento de Informática - Universidade Estadual de Maringá.

de Souza, A. C. E. (2011). Uso de animação digital para o ensino da disciplina de redes de computadores. Monografia (Graduação). Departamento de Informática - Universidade Estadual de Maringá.

Lima, J. R. d. and Tavares, O. d. L. (2015). Ambiente virtual de aprendizagem. Anais do SBIE 2015, 26:702-711.

Pansanato, L. T. E., Silva, C. E., and Rodrigues, L. (2012). Uma experiência de inclusão de estudante cego na educação superior em computação. $20^{\circ}$ WEI - Workshop sobre Educação em Computação. Curitiba, Paraná.

Santana, T. S., Ribeiro, N. C. S., and Prietch, S. S. (2009). A utilização da animação digital no processo de ensino-aprendizagem de estrutura de dados. VIII Workshop de Educação e Informática (VII WEIBASE), pages 1-9.

Santos, L. (2012). Implicações do status de nativos digitais para a relação entre gerações (professor e aluno) no contexto escolar. Olhares sobre a cibercultura, pages 131-140.

Tanamati, E. (2012). Uso de animações no apoio ao ensino da disciplina de redes de computadores. Monografia (Graduação). Departamento de Informática - Universidade Estadual de Maringá.

Torres, E. F., Mazzoni, A. A., and da Mota Alves, J. B. (2002). A acessibilidade à informação no espaço digital. Ci. Inf., 31(3):83-91.

Urquiza-Fuentes, J. and Velazquez-Iturbide, J. A. (2012). [acm press the 17th acm annual conference - haifa, israel (2012.07.03-2012.07.05)] proceedings of the 17th acm annual conference on innovation and technology in computer science education - iticse '12 comparing the effectiveness of different educational uses of program animations.

W3C (2014). Web content accessibility guidelines (wcag) 2.0. https : / / www . w3 . org/Translations/WCAG20-pt-br/WCAG20-pt-br-20141024/. Acessado em 11 de janeiro de 2016.

Wiley, D. A. (2000). Connecting learning objects to instructional design theory: a definition a metaphor, and a taxonomy. Disponível em: http://reusability.org/ read/chapters/wiley. doc. Acesso em: 05 nov. 2015. 